\title{
GESTIÓN Y CONSERVACIÓN DE LA FACHADA LITORAL EN EL ÁMBITO DEL ORDENAMIENTO INTEGRADO EN ZONAS COSTERAS
}

Ciro Jaramillo Molina*

* Ingeniero Civil de la Universidad del Valle, Especialista en Ingeniería de Transporte de la Universidad Politécnica de Valencia, España.

Profesor del Grupo de Investigación en Transporte, Tránsito y Vías - GITTV, Escuela de Ingeniería Civil y Geomática. Facultad de Ingeniería - Universidad del Valle.

\section{RESUMEN}

De manera inicial se tratan los lineamientos para la recuperación de la fachada costera y su relación con las obras de protección; las infraestructuras de transporte y de manera especial la explotación turística del litoral; posteriormente se menciona el mantenimiento y gestión del escenario costero, además de incluir los métodos de control del detrimento del paisaje provocado por la erosión, se hace mención a los métodos pasivos y activos. Todo esto dentro de lo que sería una estrategia global: la ordenación integrada de las zonas costeras. 


\begin{abstract}
The guidelines for the coastal-front recovery will be explained firstly, including its relationship with the following factors: coastline protections works, preservation of the transportation infrastructure and, primordially, a tourism development of the seabord. Then, the coastal-scene maintenance and management will be discussed, mentioning also methods for controlling landscape detrimental changes, taking into account both active and passive conservations techniques. All this included, of course, in what would be considered as a global strategy: A totally integrated regulations of all coastal areas in the world.
\end{abstract}

\section{CONSIDERACIONES GENERALES}

No cabe duda de que el momento ideal para incorporar las medidas de optimización ambiental y socioeconómica de cualquier actividad es el de su planificación. La corriente imperante ha dado ya ese paso de incorporación de las consideraciones pluridisciplinares a las decisiones en materia de planificación. En la fase de construcción, el tipo de medidas correctoras que se hayan diseñado deben ser ejecutadas simultáneamente con las obras que se traten de corregir, para que la ejecución del proyecto y su corrección sean una misma área.

Los criterios que deben presidir estas obras son: mejoras en las condiciones del paisaje extrínseco, regeneración de la cubierta vegetal modificada con especies preferentemente autóctonas, utilizar una determinada proporción entre los elementos caducos y persistentes que aumenten el valor paisajístico intrínseco de la zona, como la formación de pantallas acústicas o visuales, el diseño de técnicas de potenciación de vistas positivas y el tratamiento de espacios marginales.
Es obvio que para conseguir estos resultados deben emplearse técnicas de simulación, en las que pueden apreciarse la diferencia entre el estado final propuesto en el proyecto de una obra y el resultado que va a lograrse tras el tratamiento restaurador que le corresponde.

Cuando se finalizan las obras será necesario vigilar las tareas previstas en las recomendaciones de seguimiento y control que es necesario considerar en la fase de obsolescencia, dado que cualquier infraestructura o actividad llegará a la misma. Así debe recomendarse en general el desmonte y restauración totales de cualquier instalación auxiliar y de todo el suelo ocupado por la obra obsoleta, aplicándosele un tratamiento paisajístico complementario.

\section{LINEAMIENTOS PARA LA RECUPERACIÓN DE LA FACHADA COSTERA}

La zona costera requiere gestión para la recuperación de acuerdo a dos escalas de valores: el valor ecológico que es dirigido a conservar los ecosistemas y recursos bióticos, incluyendo el paisaje y el valor económico que exige el control adecuado de las actividades humanas hacia la explotación consumista' de la zona litoral.

\section{De los Paisajes Naturales y las Obras de Protección del Litoral}

Las acciones correctivas en arrecifes, manglares, playas, lagunas o estuario y

\footnotetext{
1 El uso irracional del recurso sigue esta secuencia: identificación del recurso, explotación del mismo, cosecha excesiva y el rápido decrecimiento productivo (contra lo elemental en cuanto a conservación se refiere).

2 Recuperación Ambiental del Arenal de Liencres, Playa de Valdearenas (España). En este proyecto se abordan las características más importantes del medio natural que compone el sistema dunar, las causas de su degradación y los métodos empleados para su regeneración.
} 
hierbas submarinas (ver figura 1), serán el resultado de una planificación costera integrada. A continuación se relacionan algunas de las estrategias para la protección de las costas:

- Desarrollar una línea de límite para la construcción de las estructuras costeras.

- Cuando se construye una obstrucción Importante para los sedimentos a lo largo del litoral, hay que dejar un paso adecuado para el transporte de arena.

- Siempre que sea posible, hágase uso de soluciones de ingeniería flexibles, en vez de soluciones inflexibles para solucionar los problemas de erosión de las playas.

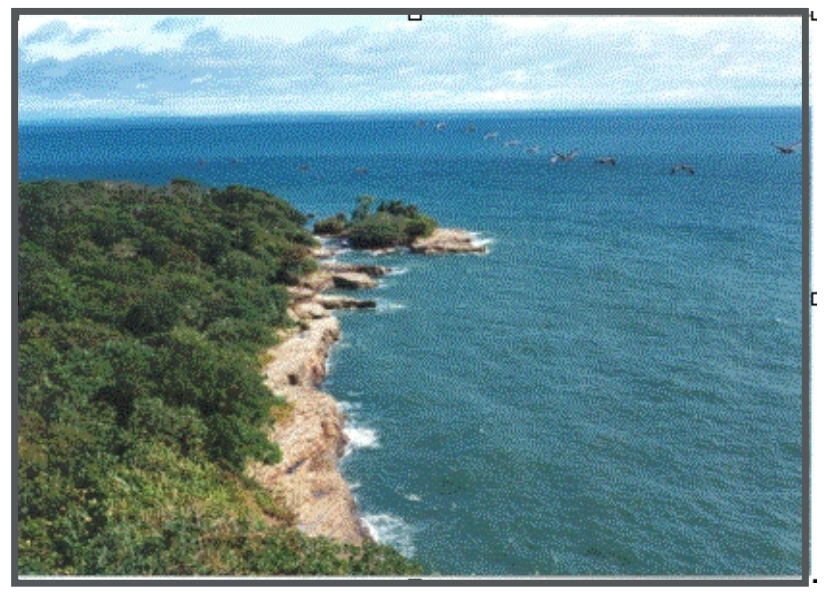

Figura 1

- No se deben destruir o modificar las dunas. Esto no permite espacio para que tengan lugar los ciclos de erosión y depósito sobre las playas. Las dunas deben ser salvaguardadas, de manera que la arena pueda moverse hacia adentro y afuera a lo largo de la playa en un ciclo natural ininterrumpido.

- Si se desea la existencia de una playa no se debe minar la arena de las dunas, playas o áreas costeras cercanas.

- No hay que entrar en pánico después de que una tormenta haya alterado drásticamente la playa. Hay que permitir que el ciclo normal de la playa regrese la arena.

- Hay que tratar de entender el sistema normal de la playa antes de que sea alterado.

- Exigir un control sobre escorrentías de aguas residuales y otras formas difusas de contaminación.

- Las prácticas de arrastre o rastreo y otras operaciones de captura pesquera deben ser modificadas.

- Los lechos de hierbas submarinas deben ser inventariados y cartografiados.

\section{De los Paisajes Relacionados con Infraestructura de Transporte}

En la zona costera la mayoría de los recursos costeros exhiben la capacidad de colonizar rápidamente aquellos hábitats que les son convenientes cerca de los puertos, atracaderos y vías acuáticas costeras (ver figura 2). Los ecosistemas costeros poseen una asombrosa habilidad natural para automantenerse y autorenovarse, cuando aquellas características básicas del hábitat que favorecieron su origen son mantenidas. Sin embargo, son extremadamente sensibles a factores que alteren dichas condiciones. Para llevar al máximo los beneficios económicos y reducir al mínimo los costos y riesgos económicos, la planificación de puertos y atracaderos deben considerar seriamente en qué forma aquellas modificaciones afectan el ambiente físico, biótico y socioeconómico del puerto y las áreas circundantes. Las actuaciones a realizar podrán ser las siguientes:

- Las mejoras en los puertos deben ser localizadas y diseñadas en tal forma que reduzcan al mínimo los cambios sobre los parámetros existentes. 
- El dragado y el relleno deben ser evitados por norma general y la eliminación de los escombros extraídos por el dragado en sitios de tierras altas es usualmente preferible.

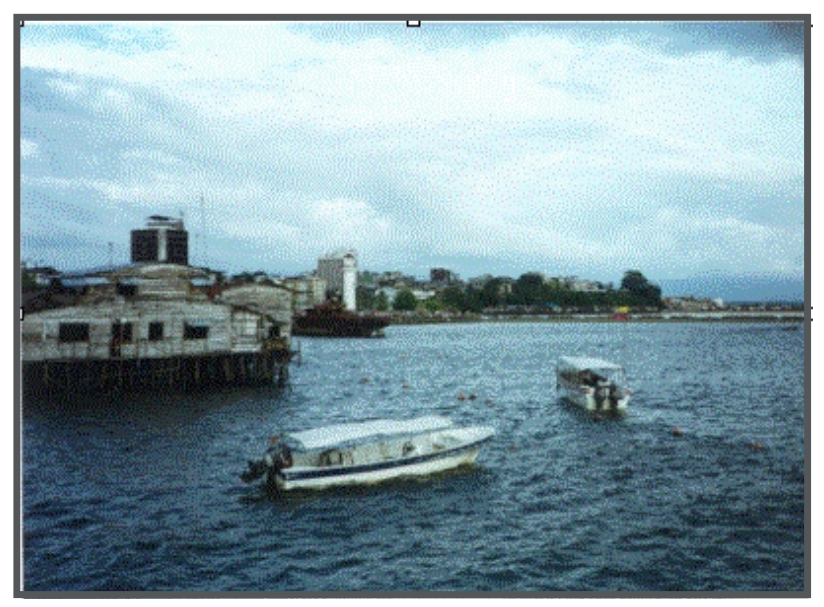

Figura 2

- Los puertos, canales y atracaderos deben mantener el equilibrio natural entre la acumulación de sedimentos y la erosión.

- Impedir el bloqueo a la circulación del agua, en particular a muelles, atracaderos, desembarcaderos o descargaderos y pilares para puentes.

- Los puertos, canales y atracaderos deben ser localizados de tal forma que evadan la presencia de recursos costeros críticos, en la mejor forma posible.

- La planificación, diseño, e ingeniería de proyectos debe ser realizado por especialistas calificados. La aplicación de un estricto profesionalismo en las normas de funcionamiento y los requisitos de diseño, puede reducir los costos económicos a largo plazo y llevar al mínimo el daño ambiental que generalmente es atribuible a prácticas inapropiadas asociadas con la construcción de carreteras, ferrovías y puentes en las tierras bajas costeras.

\section{De los Paisajes Relacionados con Turismo Costero}

Las consideraciones ambientales y paisajísticas de operación para el turismo (ver figura 3) son:

- El desarrollo del turismo costero debe estar enmarcado en el plan de desarrollo socioeconómico nacional, regional y local de forma integrada con objetivos ambientales y estrategias de desarrollo para identificar la zona más apropiada para el turismo.

- Las áreas costeras reservadas para el turismo deben ser cubiertas por planes de zonificación, primero debe realizarse un inventario que incluya el ambiente físico; el ambiente creado por el hombre; el ambiente sociocultural; y la existencia de enfermedades contagiosas.

- La capacidad de carga del área debería ser definida con el objeto de determinar la población total, sin sobrecargar la infraestructura y causar la degradación de los recursos naturales.

- Cuando sea necesario realizar desmonte, éste debe ser controlado para asegurar un impacto mínimo en los ecosistemas costeros naturales.

- Las vías de acceso deben reducir al mínimo la congestión del tráfico, los ruidos, los desechos sólidos y líquidos y otros impactos de áreas circundantes.

- El alojamiento debe ser concentrado, la escala, el tamaño y el tipo de infraestructura deben ser apropiados. Las estructuras deben ser localizadas a una buena distancia de la playa entre 100 y $300 \mathrm{~m}$.

\section{MANTENIMIENTO Y GESTIÓN DEL PAISAJE LITORAL}

Este tema es importante porque el mantenimiento y conservación del paisaje son 
vitales para el éxito del conjunto. Esto es así debido a que los materiales utilizados están vivos y crecen, experimentando cambios de día en día y de año en año. Mientras la ejecución de los proyectos de ingeniería y de edificación se termina cuando se llega al final de las obras contratadas, con el trabajo Paisajista no sucede así; el resultado obtenido constituye un conjunto joven, frágil y que tan sólo acaba de iniciar su largo período de desarrollo hacia su madurez. El que consiga o no esta madurez, de la forma prevista por el diseñador, es algo que depende del cuidado que presten al conjunto obtenido, y a lo largo de su vida, cierto número de personas.

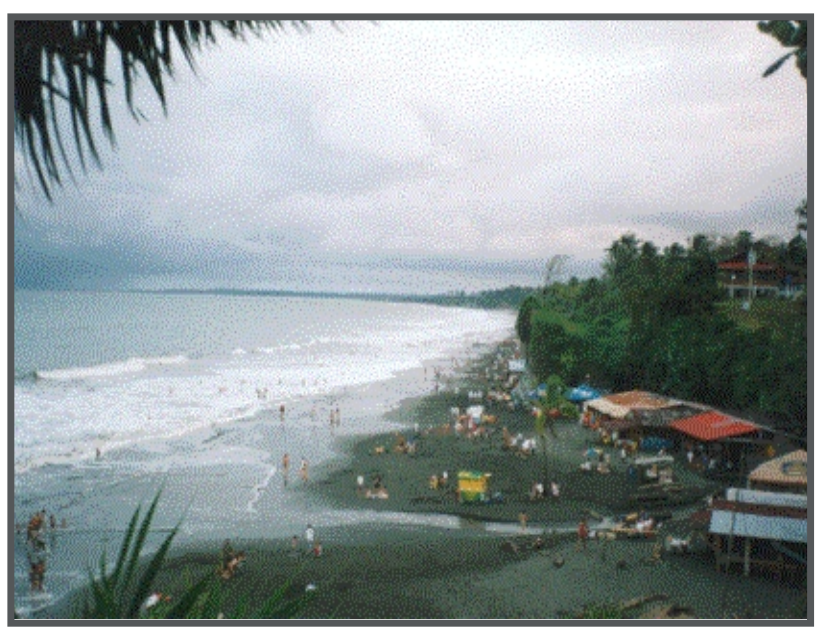

Figura 3

También es necesario distinguir entre "gestión" o administración y "mantenimiento" o conservación. La segunda de esas actividades hace referencia a las operaciones mismas que se han de realizar sobre el propio terreno y con los vegetales y plantas. La tarea administrativa, pues, corresponde a un personal calificado y con experiencia, que posea un perfecto conocimiento de lo que son las operaciones del cultivo en la horticultura.

En el planteamiento de la gestión, la escala temporal es importantísima, pues es la que explica y determina la mayor parte de los cambios que es probable que haya que introducir en el diseño original. Es una verdad incuestionable que el diseño inicial debe quedar dentro de la capacidad de mantenimiento del equipo de personal que se disponga para su conservación.

El diseñador paisajista puede contribuir al futuro mantenimiento del conjunto proporcionando un dibujo o representación gráfica donde se represente la forma en que espera que se desarrolle el paisaje proyectado cuando vaya madurando. Tal representación gráfica de conservación y mantenimiento debe ser adecuada para cada parte del paisaje, a pesar de lo cual no puede pretender el convertirse en ninguna especie de sustitutivo de la habilidad oficio de los técnicos de mantenimiento.

En el trabajo paisajista a gran escala, tanto la forma diseñada como el nivel de conservación deben aproximarse mucho más al equilibrio natural que cabe esperar para el área tratada, para reducir así la cantidad de trabajo que se requiere para lograr el fin apetecido. No obstante, el trabajo resulta muy adecuado para el tipo de tratamiento paisajista que pretende conseguir un rápido retorno a condiciones de un nuevo equilibrio ecológico que, aunque no sea idéntico al que antes existía, apunte al menos hacia un equilibrio natural y a la creación de un ecosistema que requiera un mínimo de mantenimiento. La introducción de una especie inadecuada con la idea de conseguir un efecto decorativo, si se trata de una especie invasiva, de gran tolerancia al medio capaz de escapar de su lecho, puede cambiar la pauta de vegetación para toda la zona.

El principio rector consiste en conservar la costa en el sentido de utilización de sus recursos pero sin la pérdida de sus valores y beneficios, es decir lograr la optimización 
económica del recurso costero sobre la base de la maximización de su valor natural. ${ }^{3}$ El manejo ecológico y paisajístico de la zona costera tiene como objetivo conocer la capacidad de carga de los ecosistemas costeros, con el fin de evaluar cuantitativamente los efectos adversos de las actividades humanas.

El aspecto cualitativo ${ }^{4}$ reconoce que la mayoría de las actividades de desarrollo costero con efectos negativos pueden ser absorbidas o compensadas hasta cierto grado por los procesos naturales de las aguas costeras, específicamente por el movimiento dinámico de las mareas y por el gradiente de salinidad.

El manejo costero, como la aplicación de principios ecológicos, se integra a los objetivos perseguidos por la conservación costera y particularmente al conservar la belleza escénica. Lo anterior puede ser complementado por las siguientes proposiciones: Asignar una importancia económica medible a los recursos naturales costeros y considerar posiciones económicas factibles y simples para garantizar el uso sostenido de los mismos.

Así, la utilización sostenida de un recurso significa que no puede cosecharse, extraerse o utilizarse una mayor cantidad del recurso que el producido o renovado por durante el mismo lapso de tiempo. La preservación del capital, o sea el recurso, garantizará el rendimiento, el cual estará permanentemente asegurado si no se toca el capital de base.

Así como el manejo ecológico costero está

3 Ver Snedakery Getter, 1985

4 Ibid centrado hacia la conservación de los recursos naturales y hacia el aprovechamiento racional de los mismos, el manejo costero integrado implica primordialmente la resolución de conflictos entre los diversos usos que hacen de los recursos costeros, en el sentido más amplio, es incluir además de los recursos naturales marinos y terrestres de la costa, los recursos producto del hombre, como centros urbanos y áreas de desarrollo conexas, como puertos e industrias, los recursos recreativos como playas y lugares escénicos y los recursos históricos como localidades históricas de la costa o sitios de excavaciones fósiles.

\section{MÉTODOS DE CONTROL DEL DETERIORO DEL PAISAJE PROVOCADO POR LA EROSIÓN}

En términos generales se clasifican en dos categorías; métodos estructurales y de ingeniería ${ }^{5} y$ métodos no estructurales. Estos últimos métodos son recursos de la ordenación, empleados primordialmente para controlar o aminorar los problemas ocasionados por la erosión. Estos pueden ser pasivos o activos. La mayoría de los métodos no estructurales se deben considerar como alternativas de las soluciones estructurales.

\section{Métodos Pasivos El Control del Uso de la Tierra:}

Es el permitir solamente usos que no requieran la ocupación de tierras costeras sujetas a la erosión. Toda construcción que se haga en relación con el uso permitido debe ser desmontable, o por lo menos semipermanente.

\footnotetext{
5 Para efectos del alcance de este trabajo sólo se considerarán los trabajos del tipo no estructural que tienen asociado un menor costo en la mayoría de los casos para lograr su implementación. Por ejemplo: La relación C/B social no es claramente favorable en la opción estructural.
} 


\section{Líneas de Retroceso Costero:}

Exigen que toda nueva construcción o reconstrucción de edificios e instalaciones en las tierras altas se sitúen a prudente distancia de la línea costera. Las líneas de retroceso resultan ser muy eficaces cuando se aplican a tierras costeras no desarrolladas, pero también pueden utilizarse en la planificación de la ordenación a largo plazo, exigiendo que los edificios que se vayan a reconstruir se emplacen a prudente distancia de la línea costera.

\section{Métodos Activos \\ Traslado de Estructuras Amenazadas}

El traslado a distancia prudente de la línea costera puede ser menos costoso que controlar la erosión que las amenaza.

\section{Métodos Vegetativos}

Los métodos vegetativos de control de la erosión son unos de los pocos métodos no estructurales capaces de controlar o retardar la erosión, estos métodos permiten lentificar el rítmo erosivo, aun cuando no lo detienen. Comprenden la estabilización y afirmación de las pendientes con plantaciones. Los métodos vegetativos no se emplean por sí solos en las zonas expuestas a la frecuente acción del oleaje de gran energía. En muchos casos se aplican conjuntamente con los métodos estructurales. Las tierras mojadas por las mareas o ciénagas absorben muy bien el oleaje.

\section{Corrección de la causa de la Erosión}

Las estructuras de control de la erosión, tales como escolleras y espigones pueden acelerar la erosión de las líneas costeras contiguas. La modificación de estas estructuras puede aliviar o reducir esa erosión. Siempre que sea posible, y económicamente viable, debe hacerse cuando esté al alcance para corregir - modificar el aumento de la erosión provocado por el hombre, antes de construir estructuras de control que pueden ser mucho más costosas.

\section{ASPECTOS A CONSIDERAR EN LA ELECCIÓN DE UNA TECNOLOGÍA}

Hay que proceder a una evaluación del fenómeno, así como una evaluación socioeconómica de la región afectada. El análisis costo beneficio social puede ser un instrumento muy útil en la evaluación de un proyecto de control de la erosión. La elección de una solución óptima dependerá de una evaluación de los beneficios y los costos, con arreglo a las prioridades y los criterios de los encargados de adoptar las decisiones.

\section{Beneficios:}

Los beneficios sociales de las medidas de control de la erosión pueden clasificarse ampliamente en:

\section{Financieros:}

Son los ingresos de las actividades económicas protegidos o creados por el control de la erosión, menos las partidas que puede ocasionar ese control.

Otros beneficios económicos:

Lo conforman el aumento de las oportunidades de empleo tanto en la obra misma como en las actividades económicas por ella protegidas, y el aumento de los ahorros y divisas disponibles resultante de la obra.

- Sociales: se derivan de la protección de las perturbaciones de las comunidades costeras, incluida la protección contra los peligros para la vida y la salud, así como de la participación comunitaria en la obra o en 
la preservación de las zonas de interés cultural o estético.

- Ambientales: son obvios y ya se han mencionado en apartes anteriores de este documento.

\section{Costos:}

Los costos de las medidas de control de la erosión se pueden clasificar en financieros gastos de obra- y gastos de funcionamiento. Es posible que haya cierta compensación entre los gastos de obra y los de funcionamiento. Una estructura sólidamente construida que entrañe mayores gastos de obra, puede entrañar en cambio menores gastos de conservación y reparaciones. En fin los costos financieros de una determinada medida de control de la erosión varían según el grado y la escala de la protección que ha de proporcionar esa medida.

\section{LOS PROGRAMAS DE SEGUIMIENTO Y VIGILANCIA DE LA FACHADA LITORAL}

Este aspecto es muy importante porque el mantenimiento y conservación del trabajo es imprescindible. El resultado de la obra constituye un conjunto joven, frágil y que tan sólo acaba de iniciar su largo período de desarrollo hacia la madurez.

El nivel de conservación debe acercarse mucho más al aspecto natural, cuanto más difiera el diseño de la pauta natural de vegetación, tantos más gastos y esfuerzos serán necesarios para impedir que el ecosistema siga su curso natural de sucesión ecológica:

Cada actuación podrá tener un control de

${ }^{6}$ Es altamente recomendable el seguimiento de un Plan de conservación. seguimiento y control de predicciones permitiendo el seguimiento y adecuación de las predicciones a la evolución real de la situación y su control, una vez que se ejecute la obra, y es válida tanto para métodos estructurales y de ingeniería como para métodos no estructurales.

El tipo de plantación propuesto ha de ser autosuficiente y adaptable al medio, de forma que llegue a constituir parte integrante del entorno paisajístico. No debe pues requerir cuidados especiales. La actuación humana sobre el paisaje, a largo plazo, interfiere irremediablemente con el funcionamiento del mismo.

\section{LA ORDENACIÓN INTEGRADA DE LAS ZONAS COSTERAS}

Desde hace varios decenios el medio ambiente costero y marino se ha deteriorado con rapidez, y están en peligro su integridad ecológica y la sostenibilidad de sus recursos a causa de los distintos factores como:

- El desarrollo económico, rápido y seguro.

- El aumento y el desperdicio en el consumo de recursos.

- Los conflictos que genera la asignación de recursos.

- El uso de los océanos como vertederos.

Cada vez más se presta atención a la legislación en materia del medio ambiente y al establecimiento de organismos dedicados a esta temática. Aún así, hasta hace poco tiempo no ha sido posible recoger algunos de los frutos de las iniciativas nacionales e internacionales iniciadas hace más de un decenio. La mayor parte de los intentos eran innovadores desde el punto de vista técnico y, en varios casos, han funcionado en el plano local, por lo general en zonas geográficas reducidas (ver figura 4) y/o en una gama 
limitada de actividades, pero no se han integrado de manera efectiva en el proceso de planificación del desarrollo nacional y no han atraído compromisos de aportaciones de fondos sustanciosas, por lo cual estos compromisos no han resultado tan efectivos como podrían haberlo sido a medio y a largo plazo.

Sin embargo, con la entrada en vigor de la Convención de las Naciones Unidas sobre el Derecho del Mar, y con la total conciencia de la importancia que revisten las zonas marinas para atender las necesidades humanas fundamentales en materia, por ejemplo, de alimentación, empleo, recursos, y actividades recreativas. Los países están adjudicando un nuevo papel más amplio a la ordenación integrada de los recursos marinos y costeros sujetos a jurisdicciones nacionales y a las disposiciones conexas encaminadas a la cooperación regional e internacional.

El enfoque de la ordenación se basa en un proceso dinámico de adopción de decisiones relativas a la elaboración y aplicación de estrategias. La ordenación integrada de zonas costeras no es una panacea que permite resolver todos los problemas de las zonas costeras, pero existen elementos comunes a todos los casos tales como:

- La necesidad de aplicar gradualmente la ordenación integrada de zonas costeras.

- La importancia de los recursos naturales individuales.

- El papel fundamental que desempeñan las disposiciones institucionales.

- El carácter específico y las posibilidades locales de aplicación.

- La necesidad de aplicar con flexibilidad los instrumentos y técnicas recomendados.

\section{APROXIMACIONES A ASPECTOS LEGALES DEL PAISAJE COSTERO}

Las leyes de protección de costas en algunos países europeos tipifican las infracciones en esta materia y determinan las correspondientes sanciones, así como el procedimiento para su imposición. Se intuye la ausencia de determinaciones y normas conservacionistas del paisaje y del medio. Estas expresan que la zona marítima - terrestre es un bien de dominio público estatal.

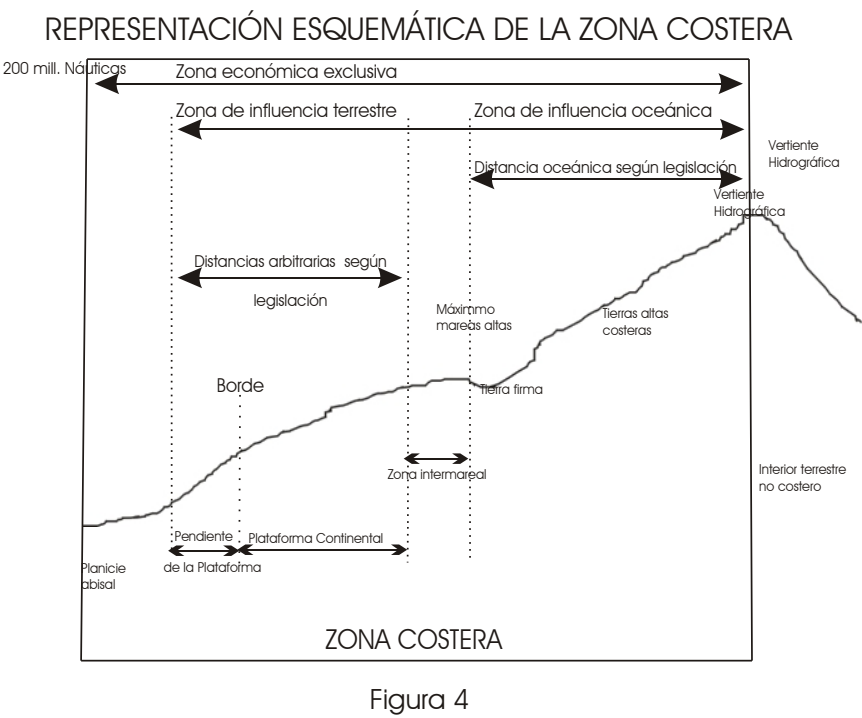

La garantía de conservación del dominio público marítimo terrestre no puede obtenerse sólo mediante una acción eficaz sobre la estrecha franja que tiene esa calificación jurídica, sino que resulta también imprescindible la actuación sobre la franja privada colindante, para evitar que la interrupción del transporte eólico de los áridos y el cierre de las perspectivas visuales para la construcción de edificaciones en pantalla, la propia sombra que proyectan los edificios sobre la ribera del mar, el vertido incontrolado y en general la incidencia negativa de la presión edificadora y de los usos y actividades que ella genera sobre el medio natural puedan causar 
daños irreparables o de muy difícil y costosa reparación. El objeto es evitar la formación de pantallas arquitectónicas en el borde de la zona de servidumbre de protección.

La costa es patrimonio colectivo especialmente valioso como espacio natural de libertad. Se debe regular la utilización de estos bienes en términos acordes con su naturaleza, sus fines y con el respeto al paisaje, al Medio Ambientey al patrimonio histórico. Son bienes de dominio publico marítimo terrestre:

- La zona marítimo terrestre: Línea de bajamarlímite hasta donde alcanzan las olas incluye: marismas esteros, etc.

- Las playas o zonas de depósito de materiales sueltos. (Dunas).

- Mar territorial y las aguas interiores.

- Los recursos naturales de la zona económica y la plataforma continental.

\section{CONSIDERACIONES FINALES}

Para un plan de manejo integral a escala nacional se deben tener como prerrequisitos de base: diagnósticos de los problemas costeros, impactos que causan las fuerzas naturales y planes de desarrollo del gobierno.

Así se deben emprender a escala nacional varias actividades relativas a la ordenación integrada de las zonas costeras, a saber:

- La preparación de documentos de planificación y gestión en determinadas zonas y como apoyo a los planes de ordenación de las zonas costeras.

- El desarrollo y aplicación de determinados instrumentos y técnicas de ordenación integrada de las zonas costeras, como por ejemplo, los sistemas de información geográfica, la evaluación de la capacidad de transporte en la esfera del turismo y la evaluación de la peligrosidad y la gestión de riesgos.

- El mejoramiento, la actualización y la ampliación de la base metodológica de la ordenación integrada de zonas costeras.

\section{BIBLIOGRAFÍA}

ALLENDE, JOSÉ. Ciudad y Territorio. Estudios Territoriales, 1995

CANTERAS, JUAN C. Y OTROS. Impacto Ambiental de Regeneración de Playas: La Playa Poniente de Gijón. Revista Ingeniería del Agua. Vol. 2 Número Extraordinario Abril de 1995.

COCCOSSIS, HARRY N. Ordenación de las Zonas Costeras Experiencia Europea. Revista de Obras Públicas. Febrero de 1988.

Curso de Medio Ambiente y Tecnología. Evaluación del Impacto Ambiental de una Obra Marítima. 1995. Universidad Politécnica de Valencia, España.

FERNÁNDEZ, PALOMA Y OTROS. Evaluación y Corrección de Impactos AmbientalesRestauración Paisajística. Instituto Geominero de España. 1991.

FERNÁNDEZ, VICTORIANOY OTROS. EI Problema de Defensa y Regeneración de Costas en España. Revista de Obras Públicas, Enero de 1988.

Gobierno Español. Ley 22 de 1988, de 28 de Julio, de Costas. 1988

La Generalitat Valenciana. Ley 2 de 1989 del 3 de Marzo de Impacto Ambiental. 1989

Ministerio del Medio Ambiente - Invemar. Primer Seminario Nacional Sobre Manejo Integrado de Zonas Costeras. 1997. 
Naciones Unidas- Departamento de Asuntos Económicos y Sociales InternacionalesSubdivisión de Economías y Tecnologías Oceánicas. Tecnologías para el Control de la Erosión Costera. 1983

Consejo Económico y Social.

Comisión Sobre el Desarrollo Sostenible. Protección de los Océanos y de los Mares de todo Tipo, Incluidos los Mares Cerrados y Semicerrados y de las Zonas Costeras y Protección, Utilización Racional y Desarrollo de sus Recursos Vivos. Abril 18 Mayo 03 de 1996.

Organización de los Estados Americanos. Convención para la Protección de la Flora, de la Fauna, y de las Bellezas Escénicas Naturales de los Países de América. Washington D.c. 1964.

PANNIER, FEDERICO. Las Costas Venezolanas: Un Reto y una Oportunidad para la Investigación, Conservación Y Gestión Ambiental. Boletín de la Academia de Ciencias Físicas, Matemáticas y Naturales ,Vol. Xlvi, Nos. 143-144. 1986.

RAMÍREZ, JOSÉ LUIS y OTRO. Recuperación Ambiental del Arenal de Liencres, Playa de Valdearenas, T.M. de Piélagos (Cantabria). 1992

SERRA, JOSÉ. Notas de Clase-Impacto Ambiental de Obras Marítimas. 1995.

SNEDAKER, SAMUEL y OTRO. Costas -Pautas para el Manejo de Recursos Costeros. Serie de Información de Recursos Renovables, National Park Service, U.S Department of the Interior. Enero 1985.

VILLEGAS, BEATRIZ Y OTROS, Deterioro $y$ Recuperación del Paisaje Litoral, Trabajo Final del Curso, 2000 - Facultad de Ingeniería Universidad del Valle. 